\title{
Percent Recovered Infinity Observed
}

National Cancer Institute

\section{Source}

National Cancer Institute. Percent Recovered Infinity Observed. NCI Thesaurus. Code C112034.

The percentage of the recovered administered dose extrapolated to infinity, calculated using the observed value of the last non-zero concentration. 